\title{
Erratum to: Lymphedema and breast cancer: a review of the literature
}

Michael Stamatakos • Charikleia Stefanaki •

Konstantinos Kontzoglou

Published online: 14 June 2011

(C) The Japanese Breast Cancer Society 2011

\section{Erratum to: Breast Cancer}

\section{DOI 10.1007/s12282-010-0246-1}

The authors' names appeared incorrectly in the article cited above. The authors' names should be correctly shown as follows:

Michael Stamatakos - Charikleia Stefanaki .

Konstantinos Kontzoglou

The online version of the original article can be found under doi:10.1007/s12282-010-0246-1.

M. Stamatakos $(\bowtie) \cdot$ C. Stefanaki

4th Department of Surgery, Medical School,

Attikon General Hospital, University of Athens,

Athens, Greece

e-mail: mixalislak@gmail.com

K. Kontzoglou

2nd Department of Propaedeutic Surgery,

Medical School, Laiko General Hospital,

University of Athens, Athens, Greece 